\title{
INTRODUCTION \\ Cushing's disease: a century of evolving diagnostics and therapeutics for an often elusive entity
}

\author{
Gabriel Zada, MD, ${ }^{1}$ John D. Carmichael, MD, ${ }^{2}$ and Warren R. Selman, MD² \\ ${ }^{1}$ Department of Neurosurgery, University of Southern California; ${ }^{2}$ Department of Medicine, Division of Endocrinology, Keck \\ School of Medicine at University of Southern California, Los Angeles, California; and ${ }^{3}$ Department of Neurological Surgery, \\ University Hospitals Case Medical Center, Cleveland, Ohio
}

It has been more than a century since Dr. Harvey Cushing saw his first patient with Cushing's disease (CD), a woman named Minnie G., in 1910. In his landmark monograph titled The Pituitary Body and its Disorders, published in 1912, Cushing described Minnie G.'s clinical situation as a "... syndrome of painful obesity, hypertrichosis, and amenorrhea, with overdevelopment of secondary sexual characteristics accompanying a low grade of hydrocephalus and increased cerebral tension. Pituitary, adrenal, pineal or ovary?"2 Cushing went on to perform a subtemporal decompression on Minnie G., resulting in temporary improvement in her headache, blood pressure, and weight gain. ${ }^{3}$ Minnie G. went on to live for several decades longer (until 1958), and her last known contact with Dr. Cushing was in 1932. Of the 12 patients with $C D$ reported in 1932 in Cushing's paper "The basophil adenomas of the pituitary body and their clinical manifestations (pituitary basophilism)," however, 8 died within a few years of their clinical presentation. ${ }^{1}$ This fact underscores the harsher reality associated with the natural history of this "malignant neuro-endocrinopathy." Namely, if persistent or left untreated, the disease can result in steady clinical decline and death.

The diagnosis and management of $\mathrm{CD}$ have evolved tremendously since Dr. Cushing's time. Elucidation of the hypothalamic-pituitary-adrenal axis and diagnostic laboratory tests, including radioimmunoassays, urinary and salivary cortisol tests, and dexamethasone suppression testing, have facilitated more and more sensitive tests for diagnosing Cushing's syndrome and CD. In the 1960s, routine incorporation of the operating microscope into transsphenoidal operations marked a new era of treatment of functional microadenomas. Magnetic resonance imaging provided another breakthrough in the management of $\mathrm{CD}$, allowing physicians to identify pituitary microadenomas, and far surpassing the diagnostic capability of lateral skull radiographs or CT imaging. The next major breakthrough in the management of CD came with inferior petrosal sinus sampling, which allowed for differentiation between central and peripheral adrenocorticotropic hormone (ACTH) secretion in MRI-negative cases, as well as the potential for detecting a gradient for laterality of secretion within the pituitary gland. Modern stereotactic radiosurgery techniques have allowed us to treat refractory CD and Nelson's syndrome, with improved outcomes. Finally, newer medical agents including pasireotide and mifepristone have shown clinical efficacy and are now available for patients with refractory $\mathrm{CD}$.

Despite these major advancements, the successful diagnosis and treatment of CD remains rife with pitfalls and challenges. In this issue of Neurosurgical Focus, a wide variety of contributions from pituitary centers of excellence around the world have been assembled to provide the latest updates, results, and opinions pertaining to this often elusive disease. This collection includes reports describing a variety of challenging diagnostic scenarios, from ectopic ACTH-secreting adenomas to cyclical CD, to adenomas in which functional expression is transformed. Results fol- 
lowing cavernous sinus and inferior petrosal sinus sampling, as well as the indications for this procedure, are presented. Updates on surgical and medical outcomes for CD, including transsphenoidal surgery and the management of refractory $\mathrm{CD}$, provide the latest summaries of clinical experience with the multidisciplinary treatment of the disease. Looking forward, a summary of gene and protein expression in ACTH-secreting adenomas may provide a glimpse of potential future strategies for drug targeting and precision medicine in patients with CD.

The combination of surgical, medical, and radiationbased treatments highlighted in this issue of Focus enables us to provide long-term remissions or cures in a majority of patients with CD. Despite this, significant work remains to further elucidate the pathophysiological mechanisms behind the disease and its high potential for recurrence, and to improve treatment for invasive and refractory CD.

http://thejns.org/doi/abs/10.3171/2014.11.FOCUS14788

\section{References}

1. Cushing H: The basophil adenomas of the pituitary body and their clinical manifestations (pituitary basophilism). Bull Johns Hopkins Hosp 50:137-195, 1932

2. Cushing H: The Pituitary Body and its Disorders: Clinical States Produced by Disorders of the Hypophysis Cerebri. Philadelphia: JB Lippincott, 1912

3. Lanzino G, Maartens NF, Laws ER Jr: Cushing's case XLV: Minnie G. J Neurosurg 97:231-234, 2002 